Focussed on Fracture and Structural Integrity

\title{
A probabilistic fatigue crack growth life approach to the definition of inspection intervals for railway axles
}

\author{
C. Mallor*, S. Calvo, J.L. Núñez, R. Rodríguez-Barrachina \\ Area of Research and Development, Instituto Tecnológico de Aragón, María de Luna, 8-50018 Zaragoza, Spain \\ cmallor@itaiinova.es, bttps:/ / orcid.org/0000-0001-8047-4211
}

\author{
A. Landaberea \\ CAF S.A., J.M. Iturrioz, 26, Beasain, Gipuzkoa, Spain
}

\begin{abstract}
Different options that rely on fracture mechanics are currently used in engineering during the design and assessment of components. One of the most important aspects is the time taken for a crack to extend to its critical size. If this time is known and it is sufficiently large, a design concept based on inspection intervals can be applied in a viable way, as is it the case of a railway axle component. To define inspection intervals that ensure the continuous and safe operation of a damage-tolerant railway axle, a reliable estimation of its fatigue crack growth life is required. Due to the uncertainties involved in the fatigue process, inspections must be devised not only considering the uncertainties in the performance of the inspection technique, but also based on a probabilistic lifespan prediction. From this premise, this paper presents a procedure for determination of inspection intervals that uses a conservative fatigue crack growth life estimation based on the lifespan probability distribution. A practical example to illustrate the reliability-based inspection planning methodology in a railway axle under random bending loading is given. The inspection intervals are further assessed in terms of overall probability of detecting cracks in successive inspections and in terms of probability of failure, considering the probability of detection curve of the non-destructive testing technique. The procedure developed provides recommendation for the definition of inspection intervals and associated inspection techniques.
\end{abstract}

KEYWORDS. Probabilistic fatigue crack growth; Damage tolerance; Inspection intervals; Probability of detection; NASGRO.

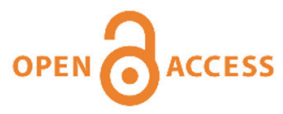

Citation: Mallor, C., Calvo, S., Núñez, J..L., Rodríguez-Barrachina, R., Landaberea, A., A probabilistic fatigue crack growth life approach to the definition of inspection intervals for railway axles, Frattura ed Integrità Strutturale, 59 (2022) 359-373.

Received: 25.10 .2021

Accepted: 22.10.2021

Published: 01.01.2022

Copyright: (C) 2020 This is an open access article under the terms of the CC-BY 4.0, which permits unrestricted use, distribution, and reproduction in any medium, provided the original author and source are credited. 


\section{INTRODUCTION}

$\mathrm{C}$ urrently, the design and operation of a railway axle is based on a two-stage safety concept comprising "safe life" and "damage tolerance analysis (DTA)" approaches [1,2]. The primary level of safety, safe life, consists in designing the axles for fatigue strength in accordance with EN 13103 standard [3] applicable for both non-powered and powered axles. The secondary level of safety, damage tolerance, relies on periodic non-destructive inspections (NDI) for crack detection, which in current practice are defined on the basis of service experience or, more recently and under development, based on fracture mechanics. The latter mainly relies on the lifespan prediction governed by the fatigue crack growth (FCG) process which is affected by many uncertainties. For instance, the experimental variability of material properties among test replications [4,5], the scattering of non-uniform loading patterns during the component operation [6,7], and the uncertainties inherent to geometrical parameters [8]. These uncertainties cause variability in the lifespan prediction and, therefore, several works propose the use of probabilistic approaches [9-13] as an alternative to deterministic ones.

Over recent years, the definition of inspection intervals in railway axles based on fracture mechanics is an active topic of research [1,14-21]. In these investigations, despite the different considerations of, initial and final crack sizes, they all use the fatigue crack growth lifespan for the subsequent inspection planning. A reliable fatigue crack growth life estimation is therefore key aspect [22]. It would thus be of interest to improve the procedures for fatigue crack growth lifespan estimation considering its stochastic nature in order to better define inspection periodicities. To obtain a probabilistic fatigue crack growth life estimation, one such interesting strategy is to construct the probability distribution of the axle lifespan as a result of the randomness of the input sources, using the Pearson distribution family based on prescribed statistical moments. This moments can be estimated by applying the full second order approach (FSOA) to the well-known fatigue crack growth NASGRO model [23] as thoroughly described in [24-26].

The levels of safety assessment for railway axles are illustrated in Fig. 1. It also includes an additional stage "In-service damage indication systems" with further options that offer potential for establishing a third stage safety concept. For completeness, the Fig. 1 also indicates the maturity of the technologies in the three stages, by using a three-level scale as follows: $(*)$ state-of-the-art; $(* *)$ present and future development; and $(* * *)$ original contribution within this research article. The figure is adapted from an extended review on safe life and damage tolerance aspects of railway axles in [1] therefore the reader is referred to this paper for full details. It is important to note that the developments in this paper aim at enriching the secondary safety level DTA, by improving the periodic inspections definition currently based on operating experience or based on a limited use of deterministic approaches using a more comprehensive probabilistic approach that provides a higher level of safety assurance. In consequence, they constitute an extension of the nowadays and under development practices by proposing the use of probabilistic fracture mechanics together with non-destructive testing methods.

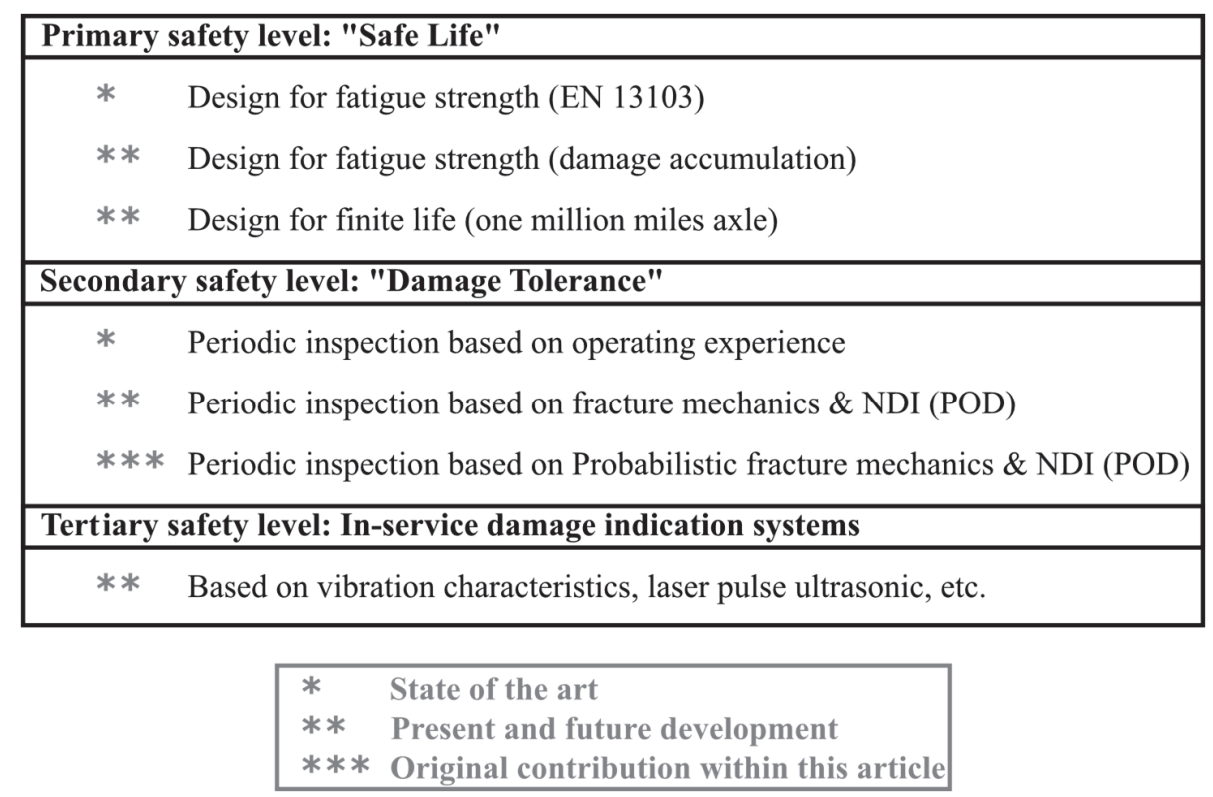

Figure 1: Components of a safety assessment system for railway axles. 
The purpose of this paper is to provide a new methodology for determination of inspection intervals in railway axles that relies on a conservative fatigue crack growth life estimation based on the lifespan probability distribution. The procedure developed extends the current state-of-the-art in damage tolerance in railway axles considering the fatigue crack growth from a probabilistic point of view. The proposed reliability-based inspection planning method is discussed through a numerical example of fatigue crack growth in a railway axle, providing recommendations for the calculation of practical inspection intervals and the associated cumulative probability of detection (CPOD) and probability of failure $\left(P_{f}\right)$ depending on the probability of detection (POD) curve of the non-destructive testing (NDT) technique.

\section{Probabilistic FATIGUE CRACK GROWTH METHODOLOGY IN THE DAMAGE TOLERANCE ASSESSMENT OF RAILWAY AXLES}

$\mathrm{T}$ he essence of damage tolerance in railway axles is to detect cracks before they become critical, providing certain level of safety for the axles in a fleet of trains by performing periodical inspections in-service. Thus, damage tolerance analyses are based on fracture mechanics to simulate crack propagation. Within the frame of the damage tolerance concept, the possibility of using probabilistic fatigue lifespan estimation is developed here. For that purpose, this section gives an overview of the steps of the damage tolerance of railway axles. Then, the propagation of uncertainty in fatigue crack growth using the FSOA and the probability distribution fit using the Pearson distribution family are outlined. Finally, the two previous elements are combined providing a reliability-based inspection interval definition.

\section{Steps of the damage tolerance analysis}

The steps of a damage tolerance analysis of a railway axle comprise [27-29]:

Step 1. establishment of the initial crack location, orientation, shape, and size,

Step 2. simulation of sub-critical crack extension, i.e., the FCG process,

Step 3. determination of critical crack size for component failure,

Step 4. determination of residual lifetime of the component, and

Step 5. establishment of inspection intervals and computation of the overall probability of crack detection.

The aim of the damage tolerance analysis in this paper is to determine inspection intervals with an associated CPOD, what is also function of the performance of the NDT method. The different steps of the analysis are explained in detail for a particular example dealing with the fatigue crack growth in a railway axle.

\section{Probabilistic fatigue crack growth life}

Starting from the assumption of an initial crack-like defect (step 1), the crack growth simulation (step 2) considers: (i) the component geometry and dimensions; (ii) the loading conditions including the bending moment (cyclic), the load spectra (in-service load sequences) and the press-fit (static); (iii) the material properties, primary the $d a / d N-\Delta K$ curve and (iv) the considered crack growth equation, commonly the NASGRO model. The NASGRO equation is shown in Eqn. (1).

$$
\frac{d a}{d \mathrm{~N}}=C\left[\left(\frac{1-f}{1-R}\right) \Delta K\right]^{n} \frac{\left(1-\frac{\Delta K_{t b}}{\Delta K}\right)^{p}}{\left(1-\frac{K_{\max }}{K_{c}}\right)^{q}}
$$

where $d a / d N$ is the crack propagation rate, $N$ is the number of applied cycles, $a$ is the crack depth, $R$ is the stress ratio, $\Delta K$ is the stress intensity factor (SIF) range and $f$ is the crack opening function, $\Delta K_{t b}$ is the threshold stress intensity factor range, $K_{c}$ is the critical stress intensity factor, and $C, n, p$, and $q$ are material empirically derived constants. It is important to note that the crack geometry is described by two parameters that represent the two axes of a semiellipse. Therefore, the crack growth rate that also depends on the boundary conditions, is calculated at two different points, the deepest point, and the crack surface point. For a more detailed description of the previous considerations please refer to [23,24].

After that, different definitions of the critical crack size (step 3) are in use. For instance, the break through the wall of the surface crack is adopted in [29], final cracks of $60 \mathrm{~mm}$ and $30 \mathrm{~mm}$ are used in different analyses in [30], and a crack depth of $20 \mathrm{~mm}$ is taken as the failure criterion in [31]. However, since the growth rate of long cracks is usually so high due to its exponential nature, the failure is imminent whatever the relatively large critical crack depth. In other words, as the critical 
crack size is selected in the unstable region, characterized by rapid, accelerating over time, unstable crack, where $K_{\max }$ asymptotically approaches $K_{c}$, the fracture is imminent, and therefore, the number of cycles calculated slightly varies when different criteria for the critical crack size are adopted. Next, the residual lifetime is calculated (step 4), that is, the number of loading cycles or the distance in kilometres, which the assumed initial crack, (step 1), would need to grow up to the final crack size, (step 3). Among all the different aspects which affect the residual lifetime (step 4), it strongly depends on the FCG process (step 2), and, as it is stochastic in nature, the residual lifetime also depends on the uncertainties inherent to the factors listed in (i) to (iv). Addressing the FCG problem from a probabilistic point of view is, therefore, a crucial point for the final (step 5), establishing inspection intervals with a probability of crack detection associated.

In order to obtain a probabilistic fatigue crack growth life estimation, this work applies a procedure that uses the first four moments of the fatigue crack growth life predicted by the FSOA to fit the parameters of a probability distribution based on the Pearson distribution family.

The FSOA for the moments of functions of random variables presented in [24] enables the prediction of the expected value and the variance of the fatigue lifespan of interest. Further extensions developed in [25] enable the prediction of the skewness and the kurtosis of the probabilistic fatigue crack growth life. The first-order second-moment (FOSM) method is the theoretical foundation of the FSOA, and, the most general equations for the expected value and covariance matrix in matrix form are presented in [26]. On this basis, the complete mathematical derivation of the FSOA for the first to fourth moments of functions of random variables using summation notation is presented in [24,25]. They present the expressions involving tensors of different orders in a simple and comprehensible way. Notice that, the first to fourth moments are related, by definition, to the expected value (first raw moment), the variance (second central moment), the skewness and the kurtosis (third and fourth central standardized moments, respectively) of the random output variable. For a detailed description, the manner in which the FSOA is applied to the fatigue crack growth NASGRO model for propagating the first to fourth moments of the fatigue life $N$ is illustrated in [24-26] through the use of the probabilistic NASGRO equations (Pr. Eqn.). Finally, the expected value of $N$, its variance, the skewness, and the kurtosis are calculated based on the first to fourth predicted moments.

At this point, the problem of fitting a probability distribution from prescribed moments arises. Commonly, the normal distribution is assumed when there is not much information available about the underlying probability distribution, notwithstanding that this assumption might not reflect the reality in some scenarios. Among the different distributions that can be considered, the Pearson distribution family is used in the methodology presented in $[25,26]$ as it is a versatile family that covers a broad range of distribution shapes. Additionally, it enables the expression of the parameters of the distribution as a function of the first four moments of the distribution without a priori hypotheses. Depending on these quantities, different common probability distributions arise, for instance, the beta, symmetrical beta, gamma, Cauchy, inverse-gamma distribution, beta prime, Student's $t$ and the normal distribution. The formulas to calculate the parameters for each type of Pearson distribution as a function of the expected value, variance, skewness and kurtosis, are enclosed in [32].

Summarizing, once the FSOA method and the Pearson family fit are applied, there is available a probabilistic description of the fatigue crack growth life, that provides relevant information about the statistical distribution of the output random variable fatigue life.

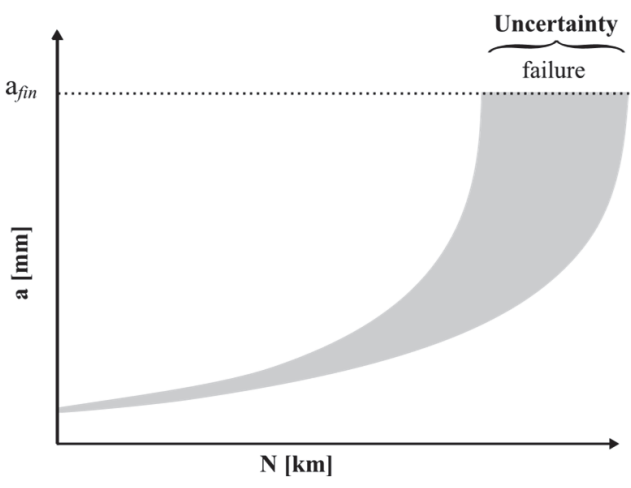

Figure 2: Probabilistic fatigue life.

\section{Reliability-based inspection interval definition}

The damage tolerance methodology overviewed in Steps of the damage tolerance analysis is commonly based on the deterministic calculation of the fatigue crack growth (step 2), but as mentioned, given the uncertainties inherent to geometric 
parameters, the variability of loads and the scatter of the material properties, the calculation of an axle lifespan should not be done with a simple deterministic calculation, and instead, a probabilistic approach is preferred. As shown in Fig. 2, the random nature of the fatigue crack growth in the railway axle needs a probabilistic description taking into account of the variabilities given by the geometric accuracy, the material properties and the actual in-service loads. With such an uncertainty, applying the probabilistic approach outlined in Probabilistic fatigue crack growth life, the probability distribution of the fatigue crack growth life is available. That is, the distribution of the fatigue life predictions with allowance for these sources of uncertainty is obtained, thus leading to an enhanced and more robust control over the safety required by these critical components.

The probability distribution can be described in various forms, such as by the survival function (SF), by the cumulative distribution function (CDF) or by the probability density function (PDF). In the context of probabilistic fatigue crack growth life in railway axles, the SF is the function that gives the probability that an axle will survive beyond any specified time, number of cycles or kilometers travelled. Frequently, in engineering, the survival function is also known as the reliability function. Alternatively, the reliability function can also be evaluated for a given reliability percent obtaining the corresponding number of kilometers travelled. In other words, in this way it provides the minimum mileage travelled for a given surviving proportion of axles. Another name for the survival function is the complementary of the cumulative distribution function (CCDF). Moreover, as it is well known, the CDF and the PDF are closely related. Given these basic premises, the working approach selects a reliability level in such a way that a conservative lifespan balancing safety and economic issues is achieved. Notice that, the input uncertainties and scatter are implicitly in the output probability distribution provided by the Pr. Eqn. and represented by its survival, cumulative distribution, and probability density functions of fatigue life. The stated procedure is illustrated in Fig. 3. As a result of the procedure, a conservative estimation of the lifespan is obtained, taking advantage of the knowledge available at the lower tail of the distribution of lives. Finally, instead of the deterministic lifespan calculation, the conservative lifespan estimation is considered as the FCG process (step 2) outcome, which is the basis for the subsequent steps oriented to the interval inspection definition.

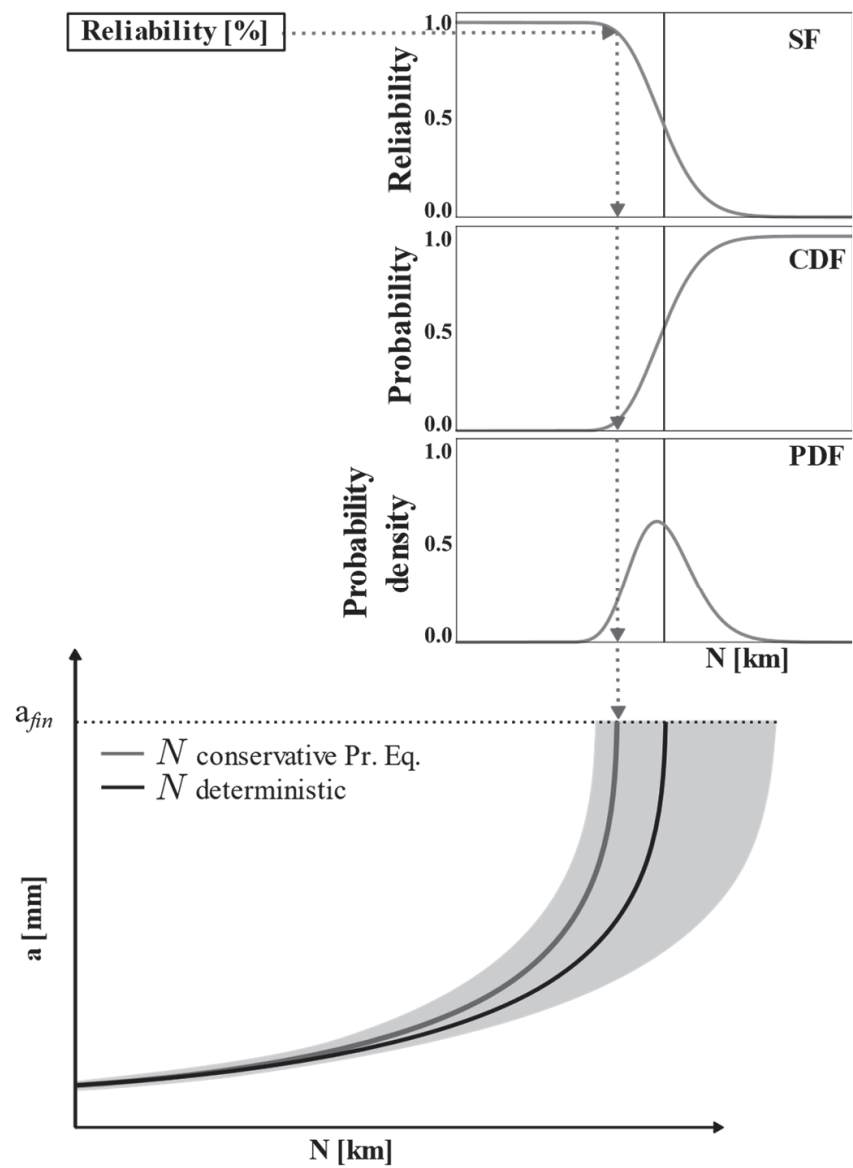

Figure 3: Conservative reliability-based life estimation from probabilistic fatigue life, illustrated by the survival function (SF), cumulative distribution function (CDF) and probability density function (PDF) of fatigue life. 
The idea for determining the periodicity of the non-destructive inspections (NDI) is depicted simply in Fig. 4. First, based on the conservative lifespan estimation, the residual lifetime (step 4) is delimited. This portion of lifetime is denoted as $N_{d e f}$ in Fig. 4 in reference to the lifetime for the definition of inspection intervals. Keeping in mind the considerations on the two-dimensional shape of the crack, and on its evolution due to fatigue loads, the $N_{d e f}$ covers the propagation from $a_{\text {min }}$ to $a_{\max }$ (steps 1 and 3), being the minimum and the maximum crack sizes considered for the lower and the higher lifetime bounds, respectively, and it is calculated through the Eq (2).

$$
N_{d e f}=N\left(a_{\max }\right)-N\left(a_{\min }\right)
$$

The usual assumption made is that $a_{\min }$ corresponds to crack size $a_{P O D} \%$ that has certain probability of being detected by NDT, for instance the crack size $a_{95 \%}$ which has a POD $=95 \%$. Finally, the inspection interval $T_{\text {ins }}$ is determined by dividing $N_{\text {def }}$ by a number of times $n_{\text {times }}$ that takes account of the number of times that the crack can be detected before a failure could occur. This simple procedure is formulated in Eq (3).

$$
T_{i n s}=\frac{N_{\text {def }}}{n_{\text {times }}}
$$

For example, the usual assumption considering $n_{\text {times }}$ equal to 2 or 3 [14], allows the crack to be observed at least twice or three times before it leads to catastrophic failure. This assumption is based on the fact that a crack could be missed at an inspection. It is, however, evident that even two or more inspections cannot ensure the crack detection.

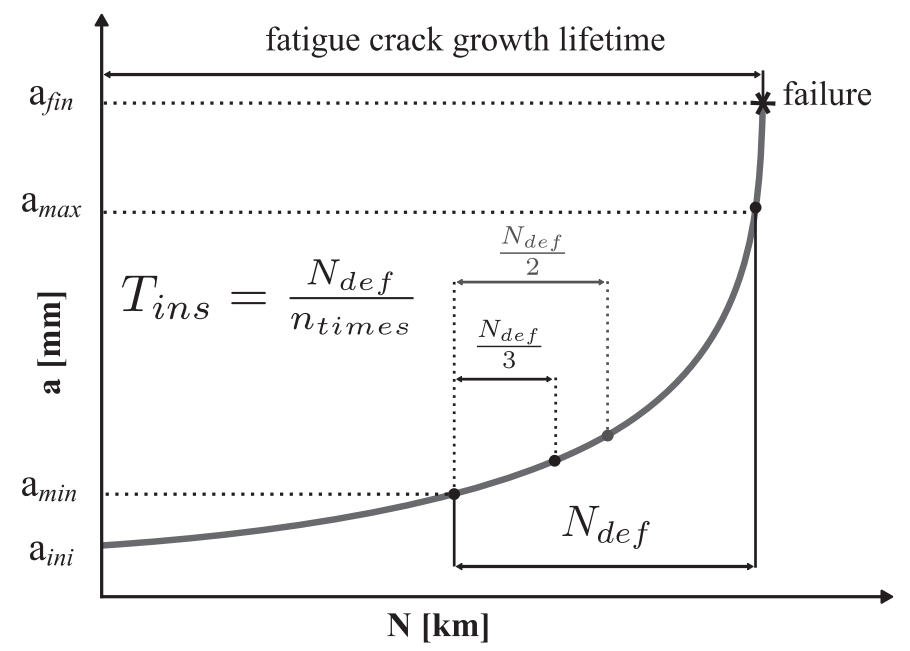

Figure 4: Calculation of the periodicity of NDT inspections, i.e., inspection intervals of maintenance.

In particular, as it is not known exactly when crack growth is triggered by an accidental event, the component will always be subjected to inspection every $T_{i n s} \mathrm{~km}$, and depending on the inspection method used, the cumulative probability of detecting (CPOD) a crack in the axle or its complementary cumulative probability of failure (CPOF) or simply referred to as probability of failure $\left(P_{f}\right)$ can be computed as described in [22]. Summarizing, the CPOD of a crack growing according to an $a-N$ curve, can be calculated based on the given number of inspections $\# i$ and the POD- $a$ curve of the NDT method used. The easiest way to calculate the CPOD is to convert the POD of the individual inspections, \#1, \#2, \#3, ..., designated with the sub-index $\# i$, to probability of non-detection (POND) by the relationship $\operatorname{POND}\left(a_{\# i}\right)=1-\operatorname{POD}\left(a_{\# i}\right)$ where a $\# i$ is the corresponding crack depth at the \# $i$ inspection. These PONDs, when multiplied give a cumulative probability of nondetection in successive inspections (CPOND). Notice, that the individual POND decreases with increasing crack length. Finally, the CPOND is converted back to its complementary CPOD. In short, the cumulative probability of detection CPOD of a crack can be evaluated as in $\mathrm{Eq}(4)$.

$$
\mathrm{CPO} D_{\# i}=1-\mathrm{CPON} D_{\# i}=1-\prod_{i} \operatorname{POND}\left(a_{\# i}\right)=1-\prod_{i}\left[1-\operatorname{POD}\left(a_{\# i}\right)\right]
$$


The probability of failure $P_{f}$, also denoted as CPOF, given that all the inspections failed to detect an actual crack, can be considered as the CPOND as expressed in Eq (5).

$$
P_{f}=C P O F_{\# i}=1-C P O D_{\# i}=C P O N D_{\# i}=\prod_{i}\left[1-P O D\left(a_{\# i}\right)\right]
$$

It is important to recall the hypothesis made here, that is, the presence of a crack (step 1) and so the probability of failure equals the probability of not detecting the crack in due time throughout the axle lifetime. This must be distinguished from the probability of failure of an arbitrary axle in a fleet of trains since an existing defect an its nucleation to a crack of that size is very unlikely. To calculate the real probability of failure, the $P_{f}$ obtained in the damage tolerance analysis should be multiplied by the probability of having a defect on the axle and by the probability that a crack will nucleate from that defect and further grow during the service life. Therefore, the real probability of failure of an axle is, by orders of magnitude, smaller than the one obtained in a damage tolerance analysis. The calculation of the real probability of failure is beyond the scope of this work. Note that, in this context, damage tolerance does not mean that a crack detected during an inspection is considered acceptable even when its size is far from being critical. In some other applications, this is a possible option, but it should be handled with care especially for safety relevant applications, as it is the case of a railway axle. In consequence, preventive maintenance is a prevailing principle in the railway industry.

In summary, the approach presented here extends the current damage tolerance principles in railway axles by means of improving the crack growth simulation (step 2), replacing the deterministic crack growth estimation by a probabilistic one. The damage tolerance assessment benefits from a better knowledge of the distribution of fatigue lifespan. As a result, it would give a more conservative recommendation for the definition of inspection intervals as it is based on a probabilistic fatigue propagation instead of on a deterministic one. The specific in-service inspection procedures shall be improved continuously in order to benefit of the continuous advance in the state-of-the-art technology.

\section{RESULTS AND DISCUSSION}

$\mathrm{T}$ his example shows the use of probabilistic fatigue life estimation in defining inspection intervals for railway axles within the frame of a damage tolerance concept. First, it uses the FSOA to obtain the expected value, variance, skewness, and kurtosis of the fatigue lifetime based on NASGRO model. Secondly, it presents the probability distribution of a particular Pearson distribution type adjusted using these first four prescribed moments. Thirdly, a conservative estimation of the lifespan is obtained based on the lifespan probability distribution. Finally, instead of the deterministic lifespan calculation, the conservative lifespan estimation is used as basis for the interval inspection definition and the subsequent CPOD calculation associated with the selected NDT technique. The methodology was applied to the example in [26]. The numerical example investigates the fatigue crack growth in the railway axle shown in Fig. 5 under random bending moment. The axle was $173 \mathrm{~mm}$ in diameter and it was made of EA1N steel defined in the EN 13261 standard [33]. A semicircular initial crack $a_{i n i}$ of $2 \mathrm{~mm}$ was postulated at the T-transition, as indicated in the cross-section of the Fig. 5. The crack grows keeping a semielliptical shape up to a final crack depth $a_{\text {fin }}$ of $50 \mathrm{~mm}$ following the direction of the radial coordinate $x$ in Fig. 5. The fatigue crack growth material parameters for the NASGRO model were those collected in [24]. Note that, the POD of a crack depends not only on the NDT technique but also on the actual crack size, as illustrated in Fig. 6 where the POD versus crack size curve for various NDT methods is shown. It is worth mentioning that ultrasonic techniques have notable different POD curves, as shown in Fig. 6, depending on the near-end or far-end application conditions pictured in Fig. 5. On the other side, magnetic particle inspections provide very good results and, also, deal with comparatively short crack depths. Based on the POD curves, the CPOD of cracks and defects in railway axles or its complementary $P_{f}$, can be computed by using the backward detection scheme described in [22].

The loads considered were the bending moment loading in the railway axle due to the vehicle weight and cargo and the press-fit loading produced by the wheel mounting with interference. The bending moment was assumed as a random input variable normally distributed with a standard deviation equal to the $1.5 \%$ of the mean value. The parameters of the bending moment distribution were: mean value $\mu_{M}=70.32[\mathrm{MN} \mathrm{mm}]$ and variance $\sigma_{M}^{2}=1.11[\mathrm{MN} \text { mm }]^{2}$. The bending moment level selected $M$ corresponded to the highest load amplitude in the spectrum of a 22.5 tonnes per axle railway, plus additional forces, generated when the train goes through curved track, over crossovers, switches, rail joints, braking efforts, etc. This assumption implied a worst case scenario since such stress level corresponded to the maximum one for axle bodies according to the EN 13103 standard [3]. Moreover, the present example considered the load spectrum acting on a railway axle over its service. The load spectrum was derived from the one available in the UIC B 169/RP 36 report [34]. The resulting service 
loading spectrum is shown in Fig. 7. In addition, the wheel was press-fitted with $0.286 \mathrm{~mm}$ interference in diameter. As a result of the spectrum combined with the randomness of the load, different number of blocks are eventually damaging, i.e., contribute to the crack growth. The reference bending stress amplitude for the mean value of bending moment and the interference stress normal to the crack surface needed for the stress intensity factor $K_{\max }$ and $K_{\min }$ evaluation were calculated via the finite element method (FEM) in [24].

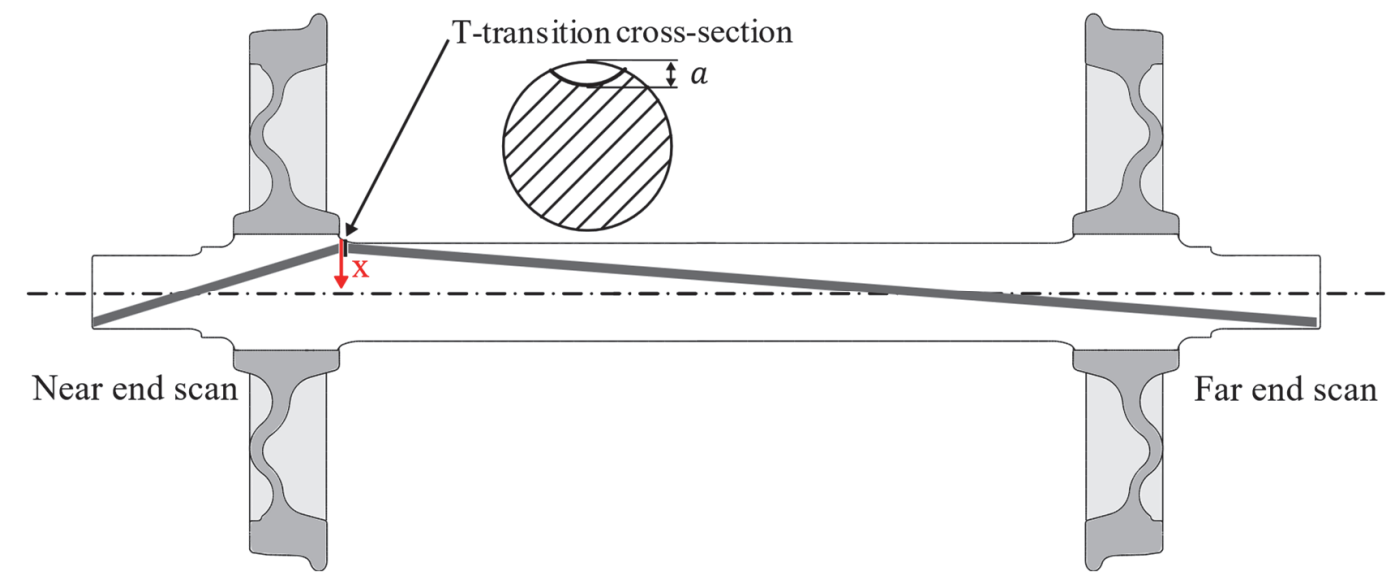

Figure 5: General view of a railway axle with a postulated crack in the T-transition inspected using near-end scan and far-end scan.

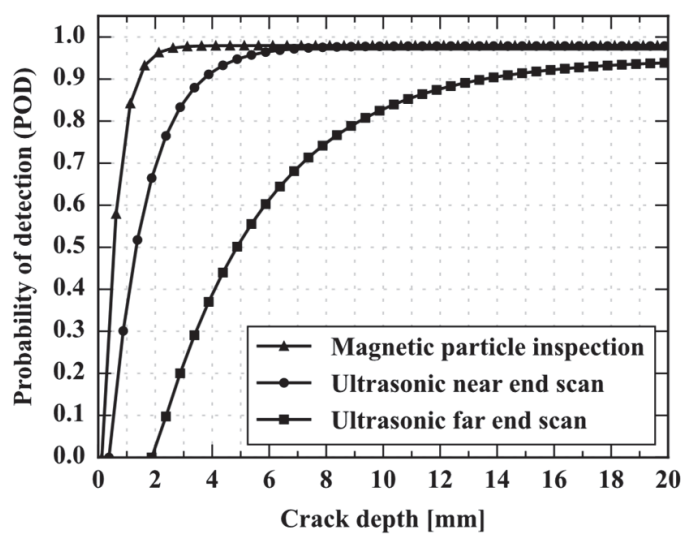

Figure 6: Probability of crack detection (POD) as a function of crack size for several non-destructive testing (NDT) methods [28].

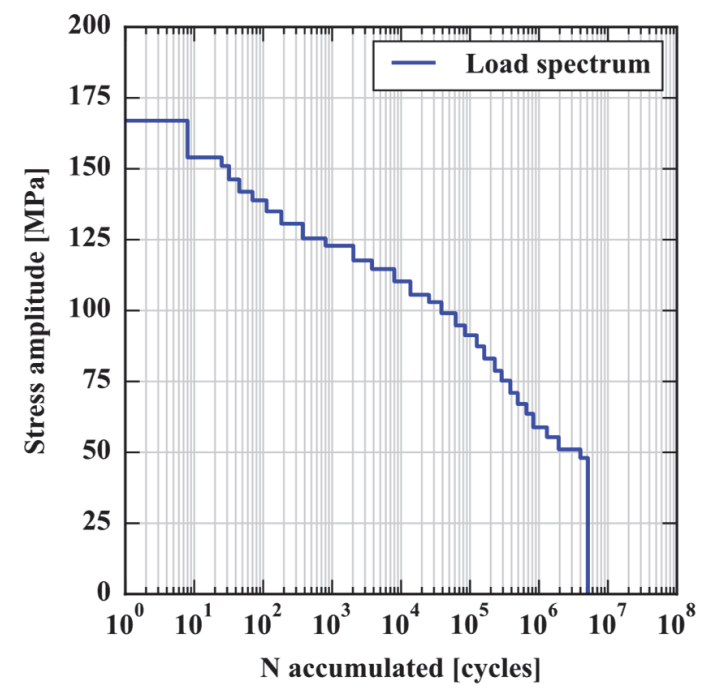

Figure 7: Stress spectrum (mileage $15000 \mathrm{~km}$ ) considered in the probabilistic analysis. 
The FSOA was applied to calculate the first moment, the second central moment, the third central moment and the fourth central moment of $d \mathrm{~N}^{i}$ at every crack depth, with the two input random variables $K_{\max }^{i}$ and $K_{\text {min }}^{i}$. Then, the expected value $\mu_{N}$, the variance $\sigma_{N}^{2}$, the skewness $\gamma_{1_{N}}$ and the kurtosis $\beta_{2}$ of the fatigue life $N$ were obtained from the $i^{t h}$ moments, providing a continuous result along the crack depth $a$. The results provided by the proposed methodology were compared with the results of 10000 Monte Carlo (MC) simulations.

To check the accuracy of the method in terms expected value $\mu_{N}$, standard deviation $\sigma_{N}$, skewness $\gamma_{1_{N}}$ and kurtosis $\beta_{2_{N}}$, the values of these moments of $N$ for a crack depth equal to $50 \mathrm{~mm}$ provided by the Monte Carlo (MC) and by the probabilistic NASGRO equations (Pr. Eqn.) using the FSOA method are gathered in Tab. 1.

\begin{tabular}{ccccc}
\hline & Units & MC & Pr. Eqn. & $\begin{array}{c}\text { Pr. Eqn.-MC } \\
\text { Error [\%] }\end{array}$ \\
$a$ & {$[\mathrm{~mm}]$} & 50 & 50 & - \\
$\mu_{N}$ & {$[\mathrm{~km}]$} & 4514673 & 4287909 & $-5.02 \%$ \\
$\sigma_{N}$ & {$[\mathrm{~km}]$} & 1402147 & 1325913 & $-5.44 \%$ \\
$\gamma_{I_{N}}$ & {$[-]$} & 1.13 & 1.10 & $-2.65 \%$ \\
$\beta_{2_{N}}$ & {$[-]$} & 5.13 & 5.38 & $4.87 \%$ \\
\hline
\end{tabular}

Table 1: Expected value, standard deviation, skewness and kurtosis of $N$ provided by Monte Carlo (MC) and by the probabilistic NASGRO equations (Pr. Eqn.).

The results demonstrated that:

- The expected value, the standard deviation or variance, the skewness and the kurtosis provided by the MC and by the probabilistic NASGRO equations are very similar, therefore, the FSOA is reasonably accurate.

- The key advantage of the FSOA method is the lower computational time, which is close to the computation time of a deterministic calculation.

- Due to the accuracy and the computational efficiency, the FSOA outperforms the conventional MC method.

At this point, the probability distribution was fitted based on the prescribed moments of the lifespan provided by the FSOA. Three scenarios were considered: (i) the lifespan was assumed to be normally distributed; (ii) the lifespan was assumed to be log-normally distributed; (iii) the Pearson distribution family was used to model the lifespan, thus avoiding the need of assuming a distribution in advance. Notice that in case (iii), the Pearson distribution type was automatically determined based on the skewness and kurtosis, leading in this example to the Pearson type VI that corresponds to the beta prime distribution. The probability density functions of the three aforementioned distributions, and the MC histogram of the fatigue life $N$ for a crack depth equal to $50 \mathrm{~mm}$ are compared in Fig. 8.

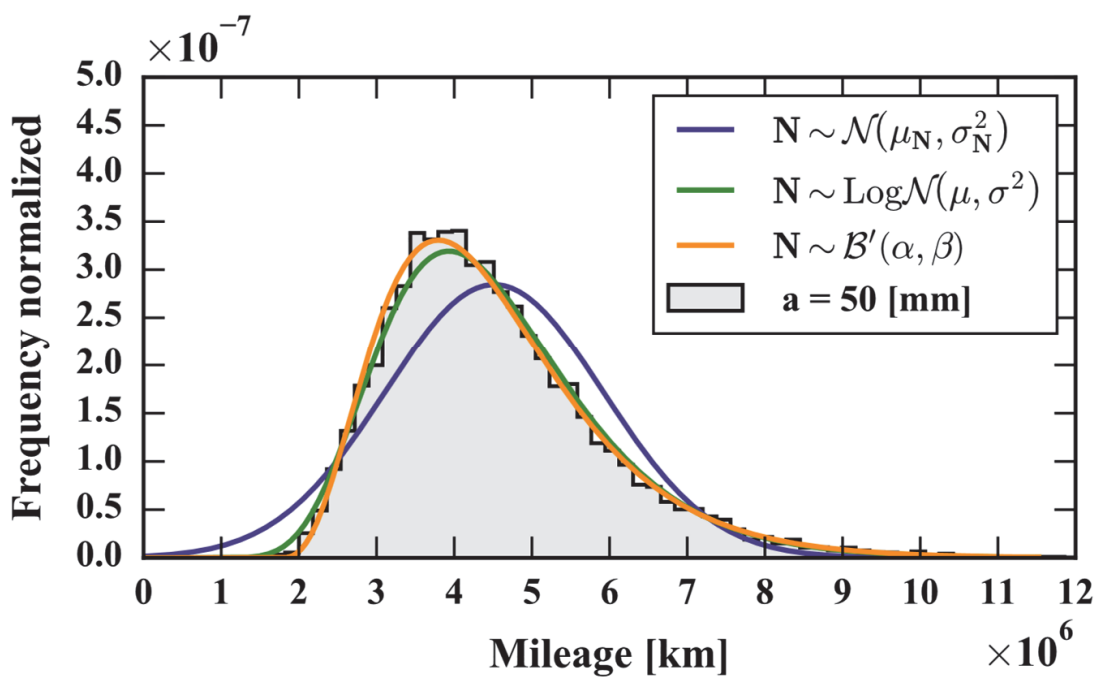

Figure 8: Histogram of fatigue life $N$ provided by the Monte Carlo (MC) and probability density function (PDF) of the normal, the log-normal and the beta prime distributions constructed from moments provided by the probabilistic NASGRO equations (Pr. Eqn.) for $50 \mathrm{~mm}$ crack depth. 
The following outcomes are achieved:

- The expected value, the variance, the skewness, and kurtosis provided by the Pr. Eqn. enable the construction of PDFs with more than two parameters as it is the case of the versatile Pearson distribution family.

- The automatic selection of the Pearson distribution type that is based on the moments of the underlying distribution is a more general procedure than the selection of an arbitrary probability distribution to fit.

- The method of moments makes calculating the parameters of the Pearson distribution type quite simple and fast.

- The similarity between the Pearson type VI, i.e., the beta prime distribution, and the MC histogram confirms that the Pearson family accurately captures and provides a good description of the underlying lifespan distribution.

- The beta prime distribution agrees well with the MC histogram for all the lifespan range, including the tails and the peak. The superiority of the beta prime distribution over the normal and the lognormal distributions to represent the MC results is clear, especially when describing the lower tail of the distribution of lives, which is of great importance in reliability and in damage tolerance assessment.

As mentioned above, the beta prime distribution of the fatigue life $N$ fitted using the FSOA, can be represented by the SF, by the CDF and by the PDF as shown in Fig. 9 for a crack depth equal to $50 \mathrm{~mm}$. The normal and the log-normal distributions are also plotted for comparative purposes.
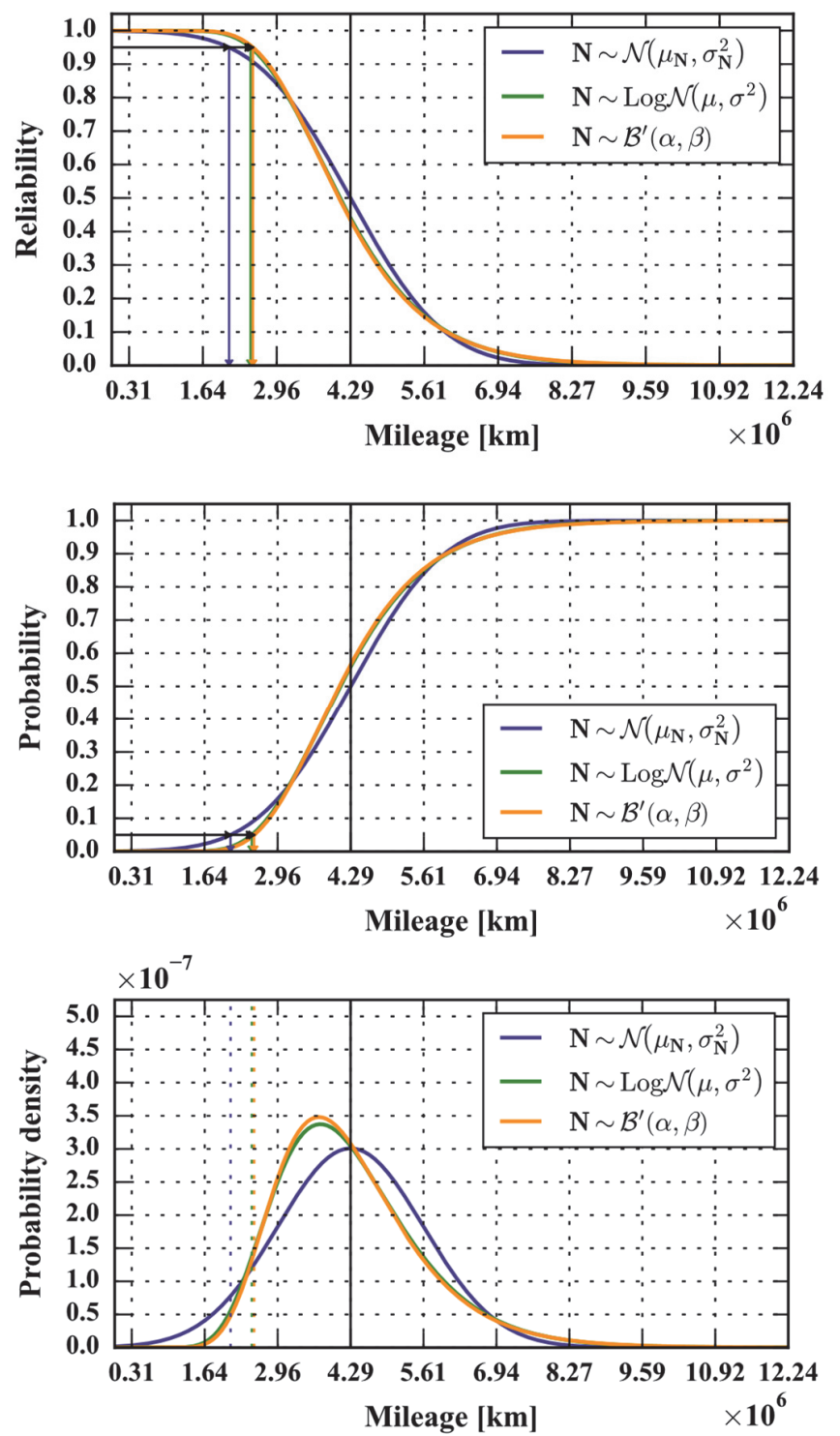

Figure 9: Survival function (SF), cumulative distribution function (CDF) and probability density function (PDF), of the normal, the log-normal and the beta prime distributions constructed from moments provided by the probabilistic NASGRO equations (Pr. Eqn.) for $50 \mathrm{~mm} \mathrm{crack}$ depth. 
The reliability-based inspection interval definition described in Reliability-based inspection interval definition, which is applied below, takes advantage of the probabilistic information contained in the Fig. 9. In order to apply the reliability-based inspection interval definition, it is necessary to briefly review the principles and requirements for the safety, serviceability and durability of structures concerning the probability of failure and reliability. The EN 1990:2002 [35] standard describes the basis for the design and verification of structures and gives guidelines for related aspects of structural reliability. It provides recommendation for the probability of failure, $P_{f}$, for structural design. It should be emphasized that these values are only notional, and therefore, do not necessarily represent the actual failure rates but, they can be used as operational values and for comparison of reliability levels of structures. As for the failure probability of a railway axle, the standard defines for a construction during the entire life, a probability of failure $P_{f, E N 1990}=7 \times 10^{-5}$. Taking this guidance, in the present case study, the probability of failure was chosen to be $7 \times 10^{-5}$ since the railway axles are non-redundant primary components whose failure consequences are extremely severe. Accordingly, the complementary reliability level is $99.993 \%$. The SF, CDF and PDF of the lifespan probability distribution determined, consider the input variabilities involved in the fatigue problem. The SF of the beta prime distribution fitted based on the FSOA moments, was evaluated for a $99.993 \%$ reliability percent, following the procedure described in Reliability-based inspection interval definition. The conservative estimation of the fatigue life based on the Pearson probability distribution for $99.993 \%$ reliability is shown in Fig. 10 .

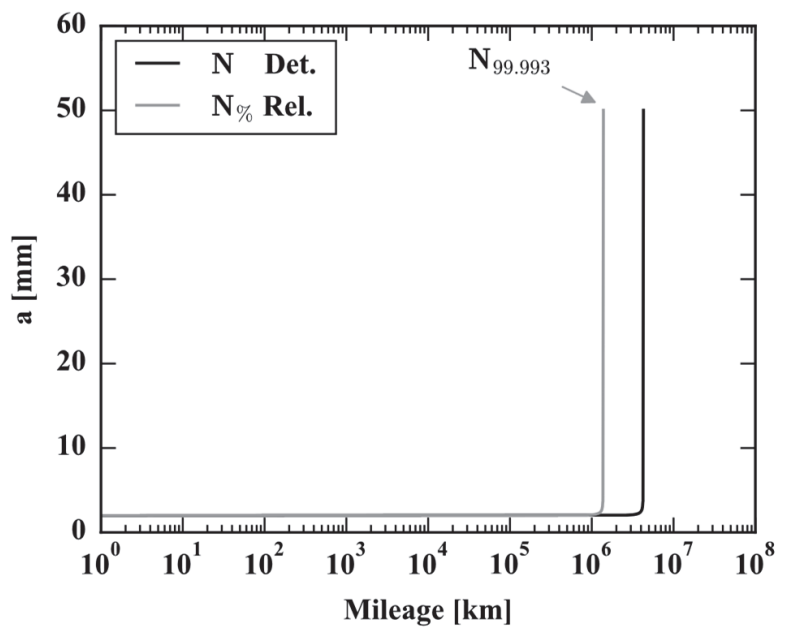

Figure 10: Estimation of a conservative number of kilometres for $99.993 \%$ reliability.

The selected proportion of axles surviving led to a minimum mileage travelled of $1.4 \times 10^{6} \mathrm{~km}$, that is, according to the probabilistic fatigue crack growth simulation, $99.993 \%$ of axles survive beyond that conservative mileage.

The calculations in Fig. 10 are conservative when compared to the deterministic estimation since there is the additional prescription of a reliability percent during the SF evaluation. It is worth noting that, apart from a reliability percent, the shape of the distribution significantly influences the conservative life estimate. Therefore, the ability of the method in describing the lower tail of the lifespan is a key aspect. Note further that because of the conservatism introduced in the adoption of a $99.993 \%$ reliability percent, the fatigue crack growth lifetime obtained from a probabilistic basis was shorter than the one obtained by simply using the deterministic calculation. This is somehow comparable to the use of a safety factor but, rather than being arbitrarily chosen, this procedure uses the available knowledge of the lifespan response as a result of the randomness of the input sources and, therefore, its application has a probabilistic foundation. In this example, the conservative lifespan calculated in this manner is obtained according to the randomness of the input loads/stresses.

Finally, the conservative lifespan estimation in Fig. 10 was considered as basis for the interval inspection definition and the for the subsequent evaluation of the CPOD and $P_{f}$ associated with the selected NDT technique. The assumptions adopted to calculate the lifetime $N_{d e f}$ (step 4) from $a_{\min }$ to $a_{\max }$ and the number of times that the crack can be detected before a failure could occur, considered for the inspection interval definition (step 5), are given in Tab. 2.

\begin{tabular}{ccc}
\hline$a_{\min }$ & $a_{\max }$ & $n_{\text {times }}$ \\
{$[\mathrm{mm}]$} & {$[\mathrm{mm}]$} & {$[-]$} \\
2 & 50 & 3 \\
\hline
\end{tabular}

Table 2: Assumptions on the inspection period definition. 
The suggested inspection interval (step 5) according to the idea depicted in Fig. 4 led to approximately $462000 \mathrm{~km}$. That is, the axle will always be subjected to periodic inspection every $T_{i n s}=462000 \mathrm{~km}$. Then, given the length of the inspection interval suggested, the history values of CPOD in successive inspections considering the backward detection scheme and the ultrasonic near-end scan NDT method are calculated according to Eq (4) and shown in Fig. 11.

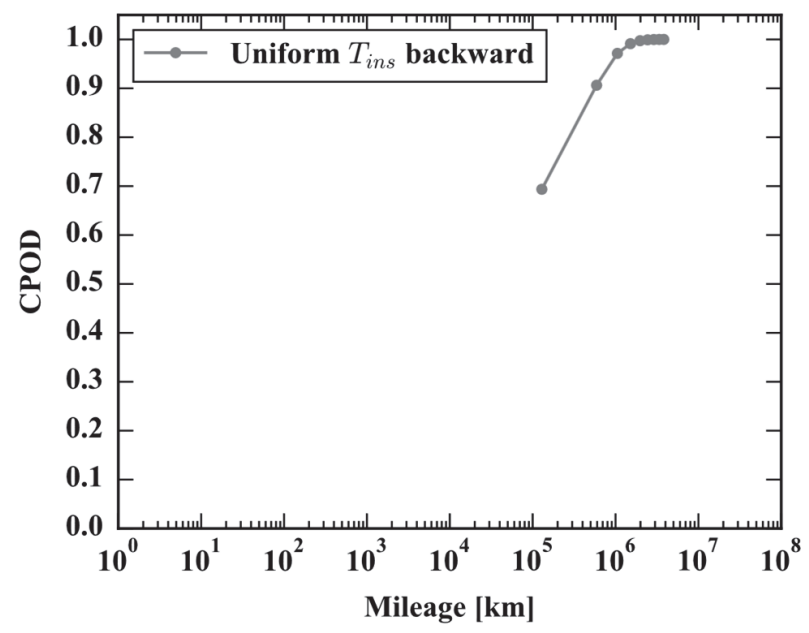

Figure 11: Cumulative probability of detection $\left(\mathrm{CPOD}_{\mathrm{i}}\right)$ considering the backward detection scheme and the near-end scan technique.

Nine inspections were possible for the span of this particular case. It can be observed an increase in instantaneous CPOD value due to the repetition inspections. Note that, the individual POD increases with increasing crack length, Fig. 6, and thus the CPOD in successive inspections becomes also higher. The results of the overall CPOD using Eq (4), and the results of $P_{f}$ using Eq (5), for the backward detection scheme and the ultrasonic near-end scan technique, are enclosed in Tab. 3.

\begin{tabular}{cccc}
\hline$T_{\text {ins }}$ & No. of ins. & Near-end scan and backward detection scheme \\
{$[\mathrm{km}]$} & {$[-]$} & CPOD & $P_{f}=1$ - CPOD \\
462000 & 9 & 0.9999844294 & $1.56 \times 10^{-5}$ \\
\hline
\end{tabular}

Table 3: CPOD and $P_{f}$ probabilities in the case of ultrasonic near-end scan technique and backwards detection scheme.

It is necessary to emphasize that the maintenance plan of railway axles for freight wagons prescribes additional off-service inspection during main wheelset overhauling, typically every $\approx 1 \times 10^{6} \mathrm{~km}$ for freight trains application. In these circumstances the inspection of axles is performed by using MPI. Performing such an inspection is not considered in the previous calculations, what would further increase the CPOD values and, accordingly, it would decrease the $P_{f}$ values.

The goal is to have a $T_{i n s}$ associated with a permissible level for probability of failure. A crucial question is what permissible probability of failure is justifiable in engineering practice. For safety critical components, as it is the case of a railway axle, a probability of failure during the entire lifetime in the order the $P_{f}$, considered in EN 1990:2002 [35] standard, $P_{f, \text { EN } 1990}=7 \times 10^{-5}$, seems reasonable. The objective in the design of the maintenance inspection plan is to achieve a $P_{f,}$ lower than the previous specific threshold. It can be observed that the $P_{f}$ for an inspection interval of $462000 \mathrm{~km}$ is compliant with the acceptable probability of failure selected as the threshold. It is demonstrated that the selected inspection intervals were adequate to ensure a high CPOD using the ultrasonic near-end scan, prior to the potential failure.

The observations of this results lead to the following general outcomes:

- The stochastic approach provides viable means for evaluating the effect of random parameters upon the definition of interval inspections within the damage tolerance concept.

- A probabilistic lifespan prediction can be integrated in the design and inspection planning of railway axles.

- The methodology devised can handle conservative fatigue crack growth estimations that are related to the input variabilities involved in the fatigue crack growth phenomenon. 
- According to the assumptions of: (i) $P_{f} \leq 7 \times 10^{-5}$; (ii) backward detection scheme; and (iii) ultrasonic near-end scan, from the results analysed, it is recommended to perform NDT using the ultrasonic near-end application condition every approximately $462000 \mathrm{~km}$.

- The procedure establishes a reliability-based inspection planning and thus, enables the optimization of maintenance expenses selecting an appropriate inspection periodicity.

\section{CONCLUSIONS}

1 his paper presents a simple procedure devised for the determination of inspection intervals within the damage tolerance analysis of railway axles, that is based on a probabilistic description of fatigue lifespan, and it is applicable for both non-powered and powered axles. It considers the input uncertainties through a conservative fatigue crack growth life estimation based on the lifespan probability distribution, benefiting from the knowledge available at the lower tail of the distribution of lives. The most important advantage of this approach is that it is based on a more conservative probabilistic rather than deterministic fatigue crack growth lifespan calculation. The benefit consists in a simple relationship between the adopted reliability in the probabilistic lifespan and the conservative prediction of the residual fatigue lifetime for practical use. Moreover, this methodology allows to focus on establishing an optimum inspection interval combining probabilistic approaches into the damage tolerance assessment phase. However, it must also balance a variety of sensitive issues of safety, economic, and vehicle availability. The probability distribution fitted from the first four prescribed moments is helpful to describe the fatigue crack growth process under stochastic conditions such as under a random bending moment loading and loading spectrum. The present approach offers potential application in practice, and it could have a remarkable effect onto the definition of inspection intervals. In the future work, the application of methodology presented will be extended, considering more parameters as random variables such as the material properties. Further investigations regarding limit state functions and conditional probability events involving fatigue failure, crack detection and reliability updating by actual observations are required. Continued efforts are needed to make reliability-based procedures and probabilistic analyses more integrated in the maintenance planning of damage-tolerant railway axles.

\section{ACKNOWLEDGEMENTS}

$\mathrm{T}$ he authors acknowledge the Spanish Ministry of Economy, Industry and Competitive through the National Programme for Research Aimed at the Challenges of Society that financially supported the project RTC-2016-4813-4.

\section{REFERENCES}

[1] Zerbst, U., Beretta, S., Köhler, G., Lawton, A., Vormwald, M., Beier, H.Th., Klinger, C., Černý, I., Rudlin, J., Heckel, T., Klingbeil, D. (2013). Safe life and damage tolerance aspects of railway axles - A review, Eng. Fract. Mech., 98, pp. 214-271. DOI: 10.1016/j.engfracmech.2012.09.029.

[2] Zerbst, U., Klinger, C., Klingbeil, D. (2013). Structural assessment of railway axles - A critical review, Eng. Fail. Anal., 35, pp. 54-65. DOI: 10.1016/j.engfailanal.2012.11.007.

[3] EN 13103-1:2017. Railway Applications. Wheelsets and bogies. Part 1: Design method for axles with external journals, European Committee for Standardization.

[4] Cervello, S. (2016). Fatigue properties of railway axles: New results of full-scale specimens from Euraxles project, Int. J. Fatigue, 86(Supplement C), pp. 2-12. DOI: 10.1016/j.ijfatigue.2015.11.028.

[5] Beretta, S., Carboni, M. (2011). Variable amplitude fatigue crack growth in a mild steel for railway axles: Experiments and predictive models, Eng. Fract. Mech., 78(5), pp. 848-862. DOI: 10.1016/j.engfracmech.2010.11.019.

[6] Pokorný, P., Náhlík, L., Hutař, P. (2014). Comparison of Different Load Spectra on Residual Fatigue Lifetime of Railway Axle, Procedia Eng., 74, pp. 313-316. DOI: 10.1016/j.proeng.2014.06.269.

[7] Pokorný, P., Hutař, P., Náhlík, L. (2016). Residual fatigue lifetime estimation of railway axles for various loading spectra, Theor. Appl. Fract. Mech., 82, pp. 25-32. DOI: 10.1016/j.tafmec.2015.06.007.

[8] Traupe, M., Landaberea, A. (2017). EURAXLES - A global approach for design, production and maintenance of railway axles: WP2 - development of numerical models for the analysis of railway axles, Mater. Werkst., 48, pp. 687-698. 
DOI: $10.1002 /$ mawe.201600570.

[9] Bea, J.A. (1997). Simulación del crecimiento de grietas por fatiga aleatoria mediante elementos probabilistas. $\mathrm{PhD}$ thesis, Universidad de Zaragoza.

[10] Bea, J.A., Doblaré, M., Gracia, L. (1999). Evaluation of the probability distribution of crack propagation life in metal fatigue by means of probabilistic finite element method and B-models, Eng. Fract. Mech., 63(6), pp. 675-711. DOI: 10.1016/S0013-7944(99)00053-3.

[11] Núñez, J.L. (2003). Análisis del fenómeno de la fatiga en metales en etapa de nucleación mediante la utilización de modelos estadísticos de daño acumulado y elementos finitos probabilistas. PhD thesis, Universidad de Zaragoza.

[12] Calvo, S. (2008). Determinación de la probabilidad de fallo en componentes métalicos sometidos a estados multiaxiales de tensión mediante la utilización de elementos finitos probabilistas y modelos estadísticos de daño acumulado. $\mathrm{PhD}$ thesis, Universidad de Zaragoza.

[13] Calvo, S., Canales, M., Gómez, C., Valdés, J.R., Núñez, J.L. (2011). Probabilistic formulation of the multiaxial fatigue damage of Liu, Int. J. Fatigue, 33(3), pp. 460-465. DOI: 10.1016/j.ijfatigue.2010.10.003.

[14] Broek, D. (1989). The Practical Use of Fracture Mechanics, Dordrecht, The Netherlands, Kluwer Academic Publishers.

[15] Beretta, S., Carboni, M., Cantini, S., Ghidini, A. (2004). Application of fatigue crack growth algorithms to railway axles and comparison of two steel grades, Proc. Inst. Mech. Eng. Part F J. Rail Rapid Transit, 218(4), pp. 317-326. DOI: $10.1243 / 0954409043125888$.

[16] Beretta, S., Carboni, M. (2006). Experiments and stochastic model for propagation lifetime of railway axles, Eng. Fract. Mech., 73(17), pp. 2627-2641. DOI: 10.1016/j.engfracmech.2006.04.024.

[17] Cocheteux, F., Pouillart, T. (2009). Design, Manufacture and Maintenance of Wheelset at SNCF, Int. J. Railw., 2(1), pp. $8-17$.

[18] Zerbst, U., Schödel, M., Beier, H.Th. (2011). Parameters affecting the damage tolerance behaviour of railway axles, Eng. Fract. Mech., 78(5), pp. 793-809. DOI: 10.1016/j.engfracmech.2010.03.013.

[19] Luke, M., Varfolomeev, I., Lütkepohl, K., Esderts, A. (2011). Fatigue crack growth in railway axles: assessment concept and validation tests, Eng. Fract. Mech., 78(5), pp. 714-730. DOI: 10.1016/j.engfracmech.2010.11.024.

[20] Mädler, K., Geburtig, T., Ullrich, D. (2016). An experimental approach to determining the residual lifetimes of wheelset axles on a full-scale wheel-rail roller test rig, Int. J. Fatigue, 86, pp. 58-63. DOI: 10.1016/j.ijfatigue.2015.06.016.

[21] Náhlík, L., Pokorný, P., Ševčík, M., Fajkoš, R., Matušek, P., Hutař, P. (2017). Fatigue lifetime estimation of railway axles, Eng. Fail. Anal., 73, pp. 139-157. DOI: 10.1016/j.engfailanal.2016.12.014.

[22] Carboni, M., Beretta, S. (2007). Effect of probability of detection upon the definition of inspection intervals for railway axles, Proc. Inst. Mech. Eng. Part F J. Rail Rapid Transit, 221(3), pp. 409-417. DOI: 10.1243/09544097JRRT132.

[23] Forman, R.G., Mettu, S.R. (1990). Behavior of surface and corner cracks subjected to tensile and bending loads in Ti6Al-4V alloy, ASTM STP 1131 Am. Soc. Test. Mater. Phila. PA, pp. 519-46.

[24] Mallor, C., Calvo, S., Núñez, J.L., Rodríguez-Barrachina, R., Landaberea, A. (2020). Full second-order approach for expected value and variance prediction of probabilistic fatigue crack growth life, Int. J. Fatigue, 133, pp. 105454. DOI: $10.1016 /$ j.ijfatigue.2019.105454.

[25] Mallor, C., Calvo, S., Núñez, J.L., Rodríguez-Barrachina, R., Landaberea, A. (2020). Uncertainty propagation using the full second-order approach for probabilistic fatigue crack growth life, Int. J. Numer. Methods Calc. Des. Eng. RIMNI, 36(3), pp. 37. DOI: $10.23967 /$ j.rimni.2020.07.004.

[26] Mallor C., Calvo S., Núñez J.L., Rodríguez-Barrachina R., Landaberea A. (2020). Propagation of uncertainty in fatigue crack growth for probabilistic life estimation, Procedia Struct. Integr., 28, pp. 619-626.

DOI: $10.1016 / j$.prostr.2020.10.072.

[27] Zerbst, U., Schwalbe, K.-H., Ainsworth, R.A. (2003). 7.01 - An Overview of Failure Assessment Methods in Codes and Standards. In: Milne, I., Ritchie, R.O., Karihaloo, B., (Eds.), Comprehensive Structural Integrity, Oxford, Pergamon, pp. 1-48.

[28] Zerbst, U., Mädler, K., Hintze, H. (2005). Fracture mechanics in railway applications - an overview, Eng. Fract. Mech., 72(2), pp. 163-194. DOI: 10.1016/j.engfracmech.2003.11.010.

[29] Zerbst, U., Vormwald, M., Andersch, C., Mädler, K., Pfuff, M. (2005). The development of a damage tolerance concept for railway components and its demonstration for a railway axle, Eng. Fract. Mech., 72(2), pp. 209-239.

DOI: 10.1016/j.engfracmech.2003.11.011.

[30] Carboni, M., Cantini, S. (2016). Advanced ultrasonic "Probability of Detection" curves for designing in-service inspection intervals, Int. J. Fatigue, 86, pp. 77-87. DOI: 10.1016/j.ijfatigue.2015.07.018.

[31] Benyon, J., Watson, A. (2001). The use of Monte-Carlo analysis to increase axle inspection interval. Proceedings of the 13th international wheelset congress, Rome, Italy, pp. 17-21. 
[32] Johnson, N.L., Kotz, S., Balakrishnan, N. (1994). Continuous univariate distributions, vol. 1-2, Wiley-Interscience.

[33] EN 13261:2009+A1:2010: Railway applications - Wheelsets and bogies - Axles - Product requirements, European Committee for Standardization.

[34] UIC B 169 RP 36:2013-12 Defect Tolerance Concept (DTC) Permitted defects and surface properties for axles under maintenance., UIC Standard.

[35] EN 1990: 2002+A1. Eurocode. Basis of structural design, European committee for standardization. 